\title{
Efficient Infrared Emission of Colloidal PbSe Nanoplatelets by Lateral Size Control
}

\author{
Lars F. Klepziga,b, Leon Biesterfelda,b, Michel Romain ${ }^{\mathrm{a}}$, André Niebur ${ }^{\mathrm{a}, \mathrm{b}}$, Anja Schlosserª, Jens Hüb- \\ ner, ${ }^{c, d}$ and Jannika Lautha,b, ${ }^{*}$ \\ a: Institute of Physical Chemistry and Electrochemistry, Leibniz Universität Hannover, Callinstr. 3A, 30167 Hannover, \\ Germany \\ b: Cluster of Excellence PhoenixD (Photonics, Optics, and Engineering - Innovation Across Disciplines), 30167 Hanno- \\ ver, Germany \\ c: Laboratory of Nano and Quantum Engineering (LNQE), Leibniz Universität Hannover, Schneiderberg 39, 30167 Han- \\ nover, Germany \\ d: Institute of Solid State Physics, Leibniz Universität Hannover, Appelstraße 2, 30167 Hannover, Germany \\ *jannika.lauth@uni-hannover.de
}

\begin{abstract}
Colloidal two-dimensional (2D) lead chalcogenide nanoplatelets (NPLs) represent highly interesting materials for near- and short wave-infrared applications including innovative glass fiber optics exhibiting negligible attenuation. In this work, we demonstrate a direct synthesis route for 2D PbSe NPLs with cubic rock salt crystal structure at low reaction temperatures of $0{ }^{\circ} \mathrm{C}$ and room temperature. A lateral size tuning of the PbSe NPLs by controlling the temperature and by adding small amounts of octylamine to the reaction leads to excitonic absorption features in the range of $800-1000 \mathrm{~nm}(1.6-1.3 \mathrm{eV})$ and narrow photoluminescence (PL) seamlessly covering the broadband infrared spectral window of $900-1450 \mathrm{~nm}(1.4-$ $0.9 \mathrm{eV}$ ). The PL quantum yield of the as-synthesized PbSe NPLs is more than doubled by a postsynthetic treatment with $\mathrm{CdCl}_{2}$ (e.g. from $14.7 \%$ to $37.4 \%$ for NPLs emitting at $980 \mathrm{~nm}$ with a FWHM of $214 \mathrm{meV}$ ). An analysis of the slightly asymmetric PL line shape of the PbSe NPLs and their characterization by ultrafast transient absorption and time-resolved PL spectroscopy reveal a surface trap related PL contribution which is successfully reduced by the $\mathrm{CdCl}_{2}$ treatment from $40 \%$ to $15 \%$. Our results open up new pathways for a direct synthesis and straightforward incorporation of colloidal PbSe NPLs as efficient infrared emitters at technologically relevant telecommunication wavelengths.
\end{abstract}

Two-dimensional (2D) IV-VI semiconductors, especially lead chalcogenide nanosheets (NSs) and nanoplatelets (NPLs), have attracted significant interest due to their promising optoelectronic properties covering the near-infrared (NIR) and shortwave-infrared (SWIR) wavelength range of the electromagnetic spectrum. ${ }^{1-3}$ The materials exhibit broadly tunable absorption and photoluminescence (PL) properties, which are controlled mainly by their quantum-confined thickness and providing access to the spectral windows needed in biological imaging or fiber optics.4-6 2D lead chalcogenide NSs for example exhibit photophysics different from their spherical nanocrystal (NC) counterparts such as strong Rashba spin-orbit coupling, ${ }^{7}$ even more efficient carrier multiplication, ${ }^{8}$ and high charge carrier mobility. 9,10 This allows for their use as active optical components for field effect transistors (FETs), ${ }^{11}$ light-emitting diodes (LEDs), ${ }^{12}$ or solar cells. ${ }^{13,14}$ Additionally, colloidal lead chalcogenides are highly interesting nanomaterials for deterministic single photon emission which is applied in photonic quantum technologies like quantum key distribution. ${ }^{15-18}$

However, synthetic access to 2D NSs and NPLs exhibiting seamlessly tunable and efficient NIR to SWIR PL is scarce. For example, Tenney et. al. synthesized HgTe NPLs decorated with HgTe NCs that exhibited efficient PL with a quantum yield (QY) of $>30 \%$ between 1000 and $1500 \mathrm{~nm}$. The synthesis is elaborate, requiring cation exchange from CdSe NPLs and a simultaneous ligand exchange. ${ }^{19}$ A similar cation exchange approach from CdSe NPLs to PbSe NPLs has been described by Galle et al.20,21 The starting CdSe NPLs show high brightness and narrow PL that is partly retained during the cation exchange. ${ }^{22}$ Thin cation exchanged PbSe NPLs from 4 monolayer (ML) CdSe yielded a PLQY of $15 \%$ centered at $1330 \mathrm{~nm}$, whereas the thickest PbSe NPLs of 6 ML exhibited a PLQY of $6 \%$ centered at $1545 \mathrm{~nm} .{ }^{20}$

While straightforward and direct colloidal synthesis methods for $\mathrm{PbS}$ and $\mathrm{PbSe} \mathrm{NCs}$ are well established, relatively few studies deal with the direct synthesis of strongly confined IV-VI semiconductor NPLs, ${ }^{2,23-28}$ even less based on PbSe. ${ }^{3}$ This could be due to the low reactivity of conventional selenium precursors including tri- $n$-alkylphosphine toward lead carboxylates, which in turn lead to the active development of selenium precursors including selenoureas. 29,30 Recently, we have shown the synthesis of ultrathin PbS NPLs with enhanced NIR emission. ${ }^{31}$

The synthesis of cubic 2D NSs and NPLs requires anisotropic crystal growth, which can be induced by different strategies including oriented attachment leading to symmetry breaking, 1,3,32 cation exchange, ${ }^{20,33}$ templated growth, ${ }^{28,34,35}$ and intrinsic instabilities leading to enhanced growth of the basal plane. ${ }^{36}$

Koh et al. describe the synthesis of thickness-controlled 2D $\mathrm{PbSe}$ NPLs by oriented attachment of PbSe NCs. ${ }^{3}$ They find a 
crucial role of halide ions to be responsible for a 2D bridging of the NCs, whereas the thickness is controlled by the amount of $\mathrm{PbI}_{2}$ added. While a tunable PL was achieved by controlling the NPL thickness, the measured PLQY of $\sim 0.1 \%$ was rather low.

Other strategies toward a Pb-based 2D crystal growth were described for perovskite NPLs. ${ }^{37}$ Almeida et al. used a mixture of primary amines and aliphatic carboxylic acid that lead to the presence of an ammonium $\left(\mathrm{RNH}_{3}{ }^{+}\right)$species in the reaction. This species increased the ionic strength of the solution and supported the growth of 2D NPLs. The enhanced solution of various precursor salts in turn lead to the controlled formation of either NCs or NPLs.

Here, we report the first direct synthesis of 2D PbSe NPLs with efficient broadband tunable infrared emission by using lead oleate and selenourea as precursors in a mixture of octylamine and oleic acid. PbSe NPLs are synthesized at room temperature or below and exhibit distinct excitonic absorption features between $800-1000 \mathrm{~nm}(1.6-1.3 \mathrm{eV})$, while narrow PL is obtained at technologically relevant telecommunication wavelengths between $900-1450 \mathrm{~nm}(1.4-0.9 \mathrm{eV}$, reaching the 0-, E- and S-band $(0.98-0.91,0.91-0.85$ and $0.85-0.81 \mathrm{eV}$, resp.). By adjusting the reaction temperature and the amount of octylamine used for the synthesis, the optical properties of the NPLs are tuned by controlling their lateral size rather than their thickness. In a subsequent step, a postsynthetic surface treatment of the PbSe NPLs with $\mathrm{CdCl}_{2}$ leads to a general doubling of the PLQY of PbSe NPLs (e.g., from $14.7 \%$ to $37.4 \%$ for NPLs emitting at $980 \mathrm{~nm}$ ), exceeding the best results for PbSe NPLs published so far.

\section{Experimental}

Chemicals. Octylamine (OcAm, $99.0 \%$ ), oleic acid (HOA, $\geq 99 \%)$ and cadmium chloride $\left(\mathrm{CdCl}_{2}, 99.99 \%\right)$ were purchased from Sigma-Aldrich/Merck. Selenourea (99.97\%) and analytical grade hexane (99.99\%, anhydrous) were purchased from Alfa Aesar. Ethanol (99.9\%, extra dry) was purchased from Acros. Tetrachloroethylene (TCE, $\geq 99.9 \%$, anhydrous) was purchased from Merck-Millipore. Octylamine was dried via freeze-pump-thawing for three times. All other reagents were used as received without further purification.

Synthesis of the lead oleate precursor $\left(\mathrm{Pb}(\mathrm{OA})_{2}\right)$. The synthesis of lead oleate is based on a method described by Hendricks et al. and has been recently proven successful for the synthesis of 2D PbS NPLs. ${ }^{31,38}$ Briefly, $5 \mathrm{~g}$ (22.4 mmol) lead oxide were stirred in $10 \mathrm{~mL}$ acetonitrile for $10 \mathrm{~min}$ at $0{ }^{\circ} \mathrm{C}$. The solution turned clear after the addition of $0.35 \mathrm{~mL}$ ( $4.54 \mathrm{mmol})$ trifluoroacetic acid and $3.1 \mathrm{~mL}(22.3 \mathrm{mmol})$ trifluoroacetic anhydride and was allowed to reach room temperature. A separate solution of $14.2 \mathrm{~mL}$ ( $45.0 \mathrm{mmol}) \mathrm{HOA}, 7.1 \mathrm{ml}$ (50.6 mmol) triethylamine and $90 \mathrm{~mL}$ isopropanol was prepared and added to the lead solution. The white precipitate was purified by recrystallization after refluxing at $80^{\circ} \mathrm{C}$ for $10 \mathrm{~min}$ until a clear solution was obtained. The solution was stored in a freezer $\left(-20^{\circ} \mathrm{C}\right)$ for $19 \mathrm{~h}$ before being filtered and washed with methanol. The dried lead oleate was stored in a glovebox at $-24^{\circ} \mathrm{C}$.

Synthesis of the selenourea precursor. In a $9 \mathrm{~mL}$ vial, a mixture of $193 \mathrm{mg}(1.57 \mathrm{mmol})$ selenourea, $2 \mathrm{~mL}$ (12mmol) OcAm, $0.2 \mathrm{~mL}(0.7 \mathrm{mmol}) \mathrm{HOA}$ and $0.75 \mathrm{~mL}$ hexane was prepared. The mixture was stirred at $35^{\circ} \mathrm{C}$ at least for $24 \mathrm{~h}$ until a clear darkorange solution was obtained. The selenium precursor was used for up to five days.

PbSe NPL synthesis. The synthesis of PbSe NPLs is based on a method by Manteiga Vázquez et al. 31 The precursor formation steps and the reaction were run under inert gas conditions either inside a glovebox or on a Schlenk apparatus. The syntheses at $0{ }^{\circ} \mathrm{C}$ were conducted using an ice bath. In a $9 \mathrm{~mL}$ vial, $182.5 \mathrm{mg}(0.3 \mathrm{mmol}) \mathrm{Pb}(\mathrm{OA})_{2}$ was dissolved in a mixture of $0.8 \mathrm{~mL}$ ( $4.9 \mathrm{mmol})$ OcAm, $0.4 \mathrm{~mL}(1.3 \mathrm{mmol}) \mathrm{HOA}$ and $1.2 \mathrm{~mL}$ ( $9.2 \mathrm{mmol}$ ) hexane. $250 \mu \mathrm{L}$ of the Se-precursor solution was injected into the $\mathrm{Pb}$-precursor solution rapidly under vigorous stirring, and was kept stirring for additional $10 \mathrm{~min}$. The solution immediately changes from colorless to dark brown upon the selenium precursor addition. The precipitation of the $\mathrm{PbSe}$ NPLs is induced by the addition of $2 \mathrm{~mL}$ dry ethanol after which the reaction product is centrifuged at $6000 \mathrm{rpm}$ for $10 \mathrm{~min}$. Precipitated PbSe NPLs are redispersed in hexane and stored in a glovebox at $-24{ }^{\circ} \mathrm{C}$. The absorption and PL properties of the PbSe NPLs can be controlled via the amount of OcAm used for the $\mathrm{Pb}(\mathrm{OA})_{2}$-precursor and the reaction temperature.

$\mathbf{C d C l}_{2}$ treatment of PbSe NPLs. For the postsynthetic $\mathrm{CdCl}_{2}$ treatment of PbSe NPLs, $0.275 \mathrm{~g}(1.5 \mathrm{mmol}) \mathrm{CdCl}_{2}$ were dissolved in a mixture of $13.5 \mathrm{~mL}(81.9 \mathrm{mmol})$ OcAm and $1.5 \mathrm{~mL}$ ( $4.7 \mathrm{mmol}$ ) HOA. $2.5 \mathrm{~mL}$ of this solution were added to the crude PbSe NPL product and stirred for additional $40 \mathrm{~min}$ at $0{ }^{\circ} \mathrm{C}$. The precipitation and storage steps were performed as described above.

Vis-NIR and PL spectroscopy. The samples for vis-NIR and PL spectroscopy were prepared by diluting the unprecipitated PbSe NPLs in TCE. Optical absorption spectra were acquired on a Cary 5000 spectrophotometer from Agilent Technologies. Steady-state PL spectra and lifetime measurements (multichannel scaling, MCS) were measured using an Edinburgh FLS 1000 UV-Vis-NIR PL spectrometer. The optical characterization of NPL dispersions was carried out using quartz cuvettes with a path length of $10 \mathrm{~mm}$. The steady-state PL was collected by exciting the samples at $500 \mathrm{~nm}$ (optical density $<0.2$ at the excitation wavelength) with a Xenon lamp and utilizing an InGaAs PMT detector. The PLQY was determined with an integrating sphere, measuring the scattering in the visible range with a 980 PMT and the PL in the NIR with the InGaAs detector of both a blank and the sample. The measured spectra were corrected for the difference in the detector sensitivities. The MCS measurements were carried out using a picosecond pulsed diode laser (EPL) with a wavelength of $445.1 \mathrm{~nm}$ and a pulse width of 110 ps by Edinburgh Instruments.

Powder X-ray diffraction (PXRD). Powder X-ray diffraction patterns were measured in Bragg-Brentano geometry using a Bruker D8 Advance equipped with $\mathrm{CuK}_{\alpha 1}$ source operating at $40 \mathrm{kV}$ and $30 \mathrm{~mA}$. The samples were prepared by drop-casting a concentrated NPL solution onto a single silicon crystal sample holder.

Transition electron microscopy (TEM). TEM analysis was conducted using a FEI Tecnai G2 F20 transmission electron microscope equipped with a field emission gun operating at $200 \mathrm{kV}$. The NPL samples for TEM were prepared by drop casting a diluted solution onto a carbon coated copper grid (300 mesh) by Quantifoil.

Energy-Dispersive X-ray Spectroscopy (EDXS). The atomic ratios of the PbSe NPL samples were determined using a scanning electron microscope (SEM) and analyzed by EDXS by using the software Oxford Instruments INCA 3000. SEM analysis was carried out using a JEOL JSM 6700F equipped with a cold field emission gun electron source. The samples were prepared by drop-casting a concentrated NPL solution onto a single silicon crystal sample holder.

Transient absorption spectroscopy (TAS). Ultrafast broadband transient optical absorption spectroscopy was performed 
on a set-up similar as described in literature. $31,39,40$ Briefly, a 100 fs laser pulse train from a Ti:sapphire amplifier system (Spectra-Physics, Spitfire ACE) centered at $800 \mathrm{~nm}$ is split $(90: 10)$ to generate a pump and a probe beam. A broadband white-light super continuum is generated by nonlinear processes inside a $\mathrm{CaF}_{2}$ or sapphire crystal $(320$ - $1600 \mathrm{~nm}$, Ultrafast, Helios FIRE) with the $10 \%$ share of the fundamental. The arrival of the probe beam with respect to the pump beam is varied by using a delay stage with a range of up to 8 ns after photoexcitation. The pump beam photoexcitation energy is
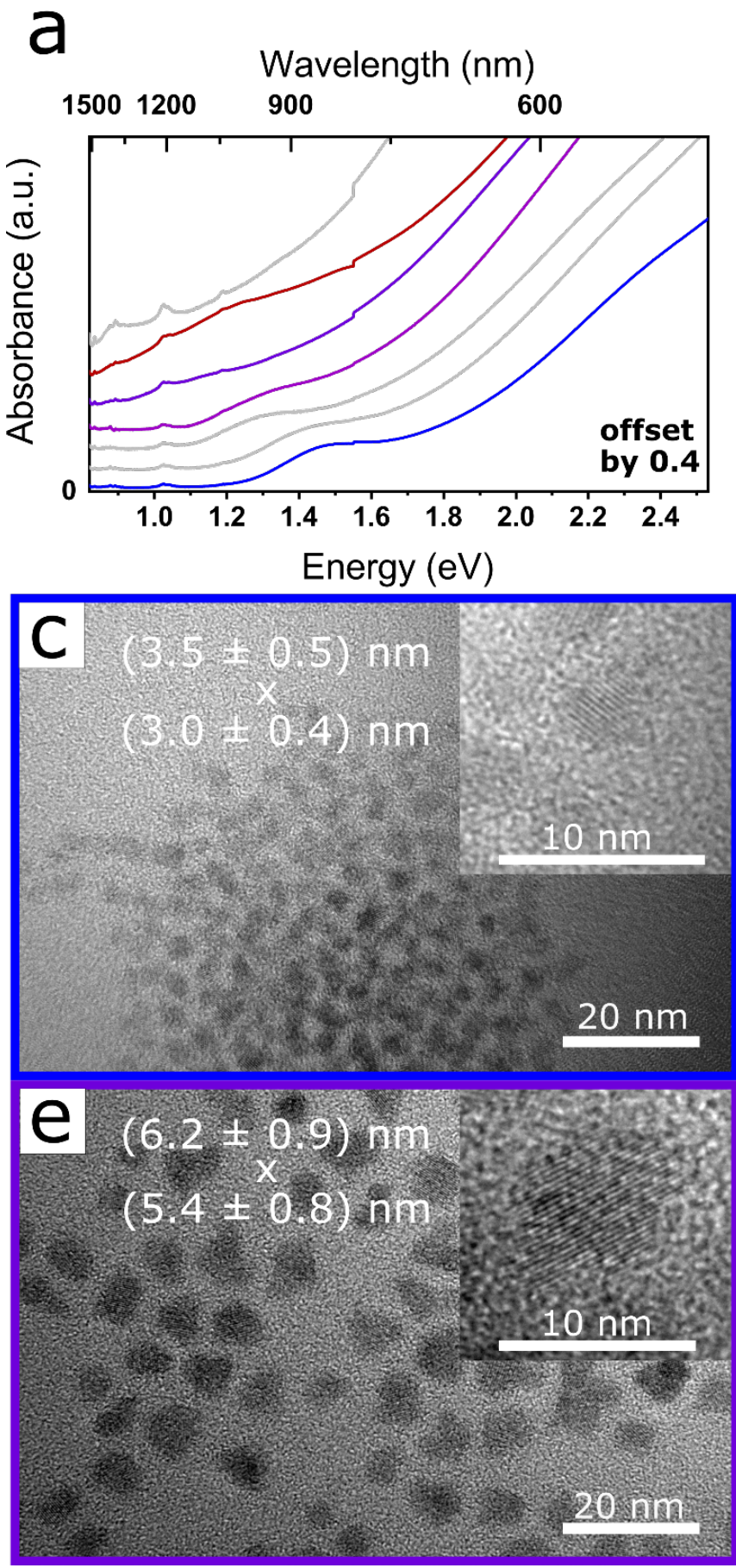

controlled by nonlinear frequency mixing in an optical parametric amplifier (OPA) system (TOPAS). The pump pulse is dumped after the photoexcitation of the sample, while the probe light is led to a fiber-coupled detector array.

For TA measurements PbSe NPL samples were diluted in TCE in $2 \mathrm{~mm}$ quartz cuvettes and kept under constant stirring to prevent photocharging. ${ }^{41}$ The optical density of the samples at the photoexcitation wavelength was kept at values $\leq 0.1$.
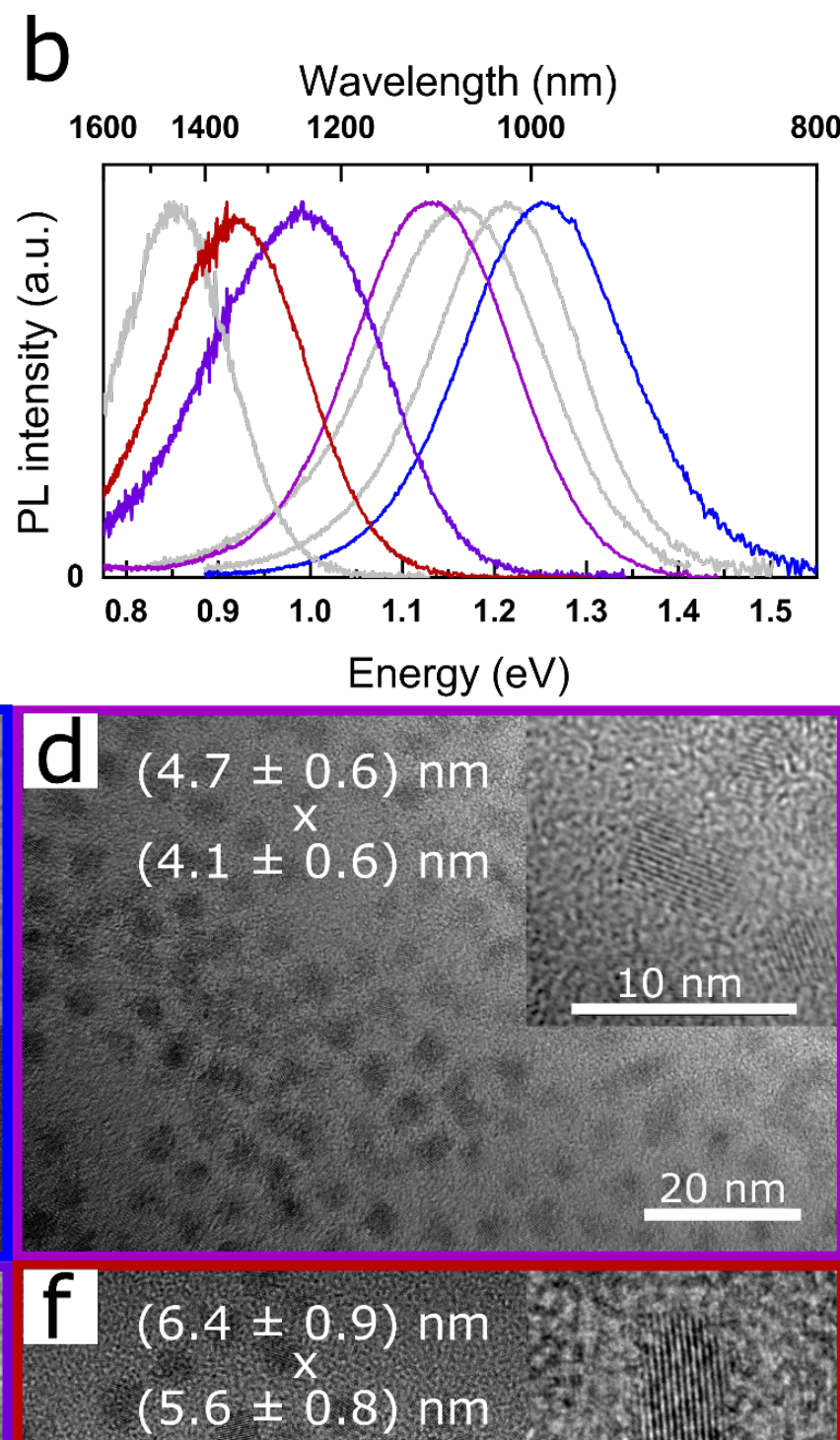

20.

Figure 1: Optical properties and TEM images of PbSe NPLs. a) Absorbance, successive spectra are plotted with an offset of 0.4 for clarity and b) associated PL of PbSe NPLs exhibiting excitonic absorption features in the range of 800 - $1000 \mathrm{~nm}$, and PL between $900-1450$ nm. c) - f) TEM images of PbSe NPLs with their associated absorption and PL shown in a) and b). PbSe NPLs exhibit a narrow size distribution, with the inset showing single crystalline NPLs in high magnification. Corresponding data sets in a) and b) 
and TEM images are color-coded. Additional properties and reaction conditions for each sample are shown in Figure 2 and listed in Table 1.

\section{Tunable absorption and PL properties of PbSe NPLS}

Figure 1a and $1 \mathrm{~b}$ shows the absorption and PL spectra of PbSe NPLs exhibiting excitonic absorption features in the range of $800-1000 \mathrm{~nm}(1.6-1.3 \mathrm{eV})$ as well as narrow PL between $900-1450 \mathrm{~nm}(1.4-0.9 \mathrm{eV})$. Figure $1 \mathrm{c}-\mathrm{f}$ depicts the corresponding TEM images of the samples, with the average NPL edge lengths given in each image (see Figure S1 for associated size histograms). PbSe NPLs exhibit a slightly rectangular shape with an aspect ratio of 1.1 to 1.2, a lateral size deviation of $15 \%$ and cubic rock salt crystal structure determined by powder XRD (see Figure S2).

We find the influence on the lateral size of the PbSe NPLs strongly dependent on the reaction temperature and the amount of OcAm used for the synthesis. While a low reaction temperature $\left(0^{\circ} \mathrm{C}\right)$ and a low amount of OcAm present during reaction is favorable for the formation of smaller PbSe NPLs, an increase of the temperature (to room temperature) and/or of the amount of OcAm leads to larger lateral NPL sizes and a tunable red shift of their absorption and PL features (see Figure 1b). An influence on the reaction kinetics, which are effected by the temperature and the strong interaction between the amine and the lead ions, likely leads to the observed spectral tunabilty.2,42 For example, Buhro and co-workers used OcAm to dissolve $\mathrm{CdX}_{2}$ precursors at room temperature, forming (CdSe) 13 nanoclusters between amine double layers, which in turn yielded 2D nanobelts upon heating. ${ }^{34} \mathrm{~A}$ similar soft template mechanism is assumed to be occurring in our case. Additionally, the interaction between OcAm and HOA leads to the formation of an ammonium species, with the equilibrium of this reaction determined by the OcAM/HOA ratio and the temperature. ${ }^{37}$ However, we did not find an influence of the amount of HOA or the ratio between HOA and OcAm on the optical properties of the obtained PbSe NPLs (see open and closed circle in Figure 2b), and consider the role of the OcAm/HOA mixture is needed in our case to ensure the complete solution of the precursors. Please note that a small amount of OcAm $(167 \mu \mathrm{l})$ and HOA $(19 \mu \mathrm{l})$ is always present during reaction due to the addition of the selenium precursor solution, and in the following description only additional amounts of OcAm and HOA added by the $\mathrm{Pb}(\mathrm{OA})_{2}$ precursor solution is stated.

By plotting the excitonic absorption of the PbSe NPLs vs. their average edge length in Figure 2a, we find the expected decreasing band gap energy with increasing NPL size. 43 The excitonic features of PbSe NPLs grow less distinct with increasing lateral NPL sizes due to an increased lateral size distribution, thus the $2^{\text {nd }}$ derivate of the absorption was used for the plot as is described in the Supporting Info (see Figure S3). Due to their slightly irregular shape, PbSe NPLs lack stacking (see Figure 1). Generally, an increase in the absorption width is found in larger NPLs as the absorption and PL is influenced by the lateral size of the NPLs rather than their thickness. We compare the obtained values with literature data for PbSe NCs expressed by an empirical sizing curve by Dai et al. ${ }^{44}$ shown in Figure 2a,

$\Delta E=4.41 /\left(D_{N C S}+0.51\right)$

with $\mathrm{D}_{\mathrm{NC}}$ as the average $\mathrm{PbSe} \mathrm{NC}$ diameter, and $\Delta E$ as the first excitonic absorption feature of the corresponding sample.

Clearly evident, we find the values for PbSe NPLs with a certain lateral size diverging toward higher energies than PbSe NCs with comparable diameters, which underpins the confinement in the thickness of the NPLs. The hyperbolic empirical fit for
PbSe NCs is explained by the highly nonparabolic curvature of the conduction and valence band near the $L$ point in lead chalcogenides and shows good agreement with both lead chalcogenide NCs and NPLs.3,44-47

We adopt a model by Kasowski et al. ${ }^{48}$ and fit our data according to

$\Delta E=\left(E_{g}^{2}+2 \hbar^{2} E_{g}\left(\pi / 0.5 D_{N P L S}\right)^{2} / m^{*}\right)^{1 / 2}$

with $E_{g}$, the bandgap of bulk PbSe $(0.278 \mathrm{eV})^{49}, D_{N P L S}$ the average NPL edge length and $m^{*}$ the effective mass of the charge carriers $\left(m^{*}=m_{e}^{*}=m_{h}^{*}\right)$. An effective mass of $m^{*}=$ $0.060 \pm 0.002 m_{e}$ exhibits the best fit in good agreement with literature values for PbSe NPLs reporting an effective mass of $0.077 m_{e}{ }^{3}$ While the lateral size of the PbSe NPLs is averaged over the width and length of the NPLs for this fit, we find the higher energy for the NPLs in comparison to spherical PbSe NCs of comparable diameter also occurs when only plotting the longer or the shorter lateral edge length (see Figure S4).
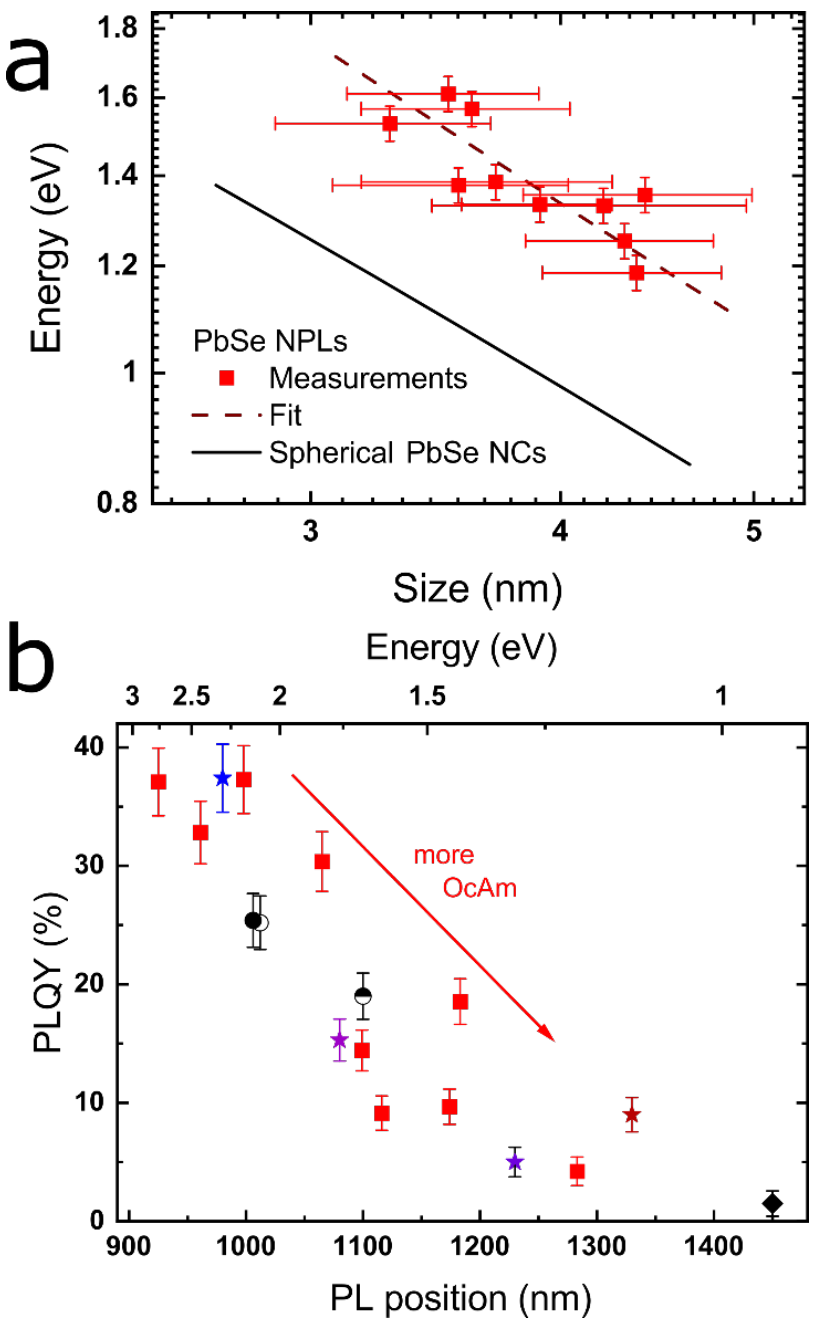

Figure 2: Influence of the reaction conditions on the PbSe NPL excitonic absorption features and PL. a) Correlation between the position of the first excitonic transition and the lateral NPL size averaged over their $\mathrm{x}$ - and $\mathrm{y}$-dimension as determined by the TEM images. The black line is an empirical fit for PbSe NCs 
published by Dai et al,44 while the dashed red line represents the fit to the PbSe NPLs and with the NPLs exhibiting considerable additional confinement due to their 2D structure. b) PLQY and PL position of different PbSe NPLs with red squares representing a reaction series with increasing OcAm amounts, while retaining the total reaction volume as well as all other synthesis parameters. The results of the different synthesis conditions are represented by varying symbols and colors shown in Table 1.

While lateral confinement has been observed in CdSe and $\mathrm{PbS}$ $\mathrm{NPLs},{ }^{28,50,51}$ its influence appears significantly higher in $\mathrm{PbSe}$ NPLs described here, which is shown in the NPL PL wich is tunable over a large spectral range (see Figure 1b) by a rather small lateral size increases of the NPLs. This originates from the larger exciton-Bohr-radius of $\mathrm{PbSe}(46 \mathrm{~nm})$ in comparison to the exciton-Bohr-radii of $\mathrm{PbS}(23 \mathrm{~nm})$ or CdSe $(5.8 \mathrm{~nm}) .52$ When comparing the absorption and PL values of our NPLs to PbSe NPLs obtained by cation exchange and oriented attachment previously, we estimate a thickness of our NPLs in the range of 1-2 nm (2-3 ML). 3,20

The PL maximum and the PLQY of $\mathrm{CdCl}_{2}$ treated samples synthesized under different reaction conditions are shown in Figure $2 \mathrm{~b}$ and summarized in Table 1. Low amounts of OcAm are sufficient for the successful synthesis of NPLs and result in the formation of the smallest NPLs. While it is possible to obtain $\mathrm{PbSe}$ NPLs without $\mathrm{OcAm}$ addition to the $\mathrm{Pb}(\mathrm{OA})_{2}$ precursor, we found the highest PLQY of $37.1 \%$ for NPLs with $0.2 \mathrm{ml}$ OcAm added during the synthesis (see Figure $1 \mathrm{c}$ and $2 \mathrm{~b}$, blue star). With increasing amount of OcAm present in the reaction, the PLQY decreases and the absorption and PL of PbSe NPLs exhibit a bathochromic shift (see Figure 2b). The colored stars in Figure $2 \mathrm{~b}$ correspond to the samples shown in Figure 1, with associated amounts of $0.2,1.2,1.4$ and $1.9 \mathrm{ml}$ OcAm present during reaction.

Table 1: PL maxima and reaction conditions for different PbSe NPL syntheses. The symbols in the first column correspond to the data shown in Figure $2 b$ ) with the first four rows corresponding to the PbSe NPLs discussed in Figure 1.

\begin{tabular}{lllllll}
\hline $\begin{array}{l}\text { PL maxi- } \\
\text { mum }(\mathrm{nm})\end{array}$ & $\begin{array}{l}\text { PLQY } \\
(\%)\end{array}$ & $\begin{array}{l}\text { OcAm } \\
(\mathrm{mL})\end{array}$ & $\begin{array}{l}\mathrm{HOA} \\
(\mathrm{mL})\end{array}$ & $\begin{array}{l}\mathrm{Hex} \\
(\mathrm{mL})\end{array}$ & $\begin{array}{l}\mathrm{T} \\
\left({ }^{\circ} \mathrm{C}\right)\end{array}$ \\
\hline$\star$ & 980 & 37.4 & 0.2 & 0.4 & 1.8 & 0 \\
$\star$ & 1080 & 15.3 & 1.2 & 0.4 & 0.8 & 0 \\
$\star$ & 1230 & 5.0 & 1.4 & 0.4 & 0.6 & 23 \\
$\star$ & 1330 & 9.0 & 1.9 & 0.5 & 1.2 & 23 \\
$\bullet$ & 1450 & 1.5 & 1.8 & 0.4 & 0.2 & 35 \\
$\ominus$ & 1100 & 19.0 & 0.2 & 0.4 & 1.8 & 23 \\
$\bigcirc$ & 1012 & 25.2 & 0.8 & 0.7 & 0.9 & 0 \\
$\bullet$ & 1006 & 25.4 & 0.8 & 0.1 & 1.5 & 0
\end{tabular}

We find the lowest temperature feasible for the NPL synthesis at $-8^{\circ} \mathrm{C}$ since lower temperatures lead to freezing of the solvent. With temperatures at or slightly above room temperature, we obtain NPLs with excitonic absorption and emission reaching up to the telecommunication 0 - and S-band (1260 $1360 \mathrm{~nm}, 0.98-0.91 \mathrm{eV}$ and $1460-1530 \mathrm{~nm}, 0.84-0.81 \mathrm{eV}$, respectively). An example for an increase in reaction temperature with all other parameters kept constant is shown in Figure $2 \mathrm{~b}$ (blue star $\left[0^{\circ} \mathrm{C}\right]$ and semicircle $\left[23^{\circ} \mathrm{C}\right]$ ). The PL maximum shifts bathochromically from $980 \mathrm{~nm}$ to $1100 \mathrm{~nm}$ with associated PLQY values of $37.4 \%$, resp. $19.0 \%$. We observe the same trend when increasing the OcAm amount in the synthesis indicating that higher PLQY values are correlated with a smaller lateral NPL size. PbSe NPLs with the smallest size (and size distribution) are obtained at reaction temperatures of $0{ }^{\circ} \mathrm{C}$, which we kept as temperature for PbSe NPLs used in the benchmark experiments in this work.

The correlation between the increasing lateral NPL size and decreasing PLQY is attributed to the formation of more surface defects in larger NPLs, which e.g. could arise as undercoordinated selenium on the NPL surface. ${ }^{51}$ Quenching sites can also be partly situated in the plane of the NPLs and be caused by missing metal atoms in a cation-rich surface. 53

\section{Postsynthetic $\mathrm{CdCl}_{2}$ treatment and time-resolved spectro- scopic investigation of PbSe NPLs}

A drastic increase of the PbSe NPL PLQY is apparent after their postsynthetic treatment with $\mathrm{CdCl}_{2}$, which has been shown recently for NPLs and NCs. ${ }^{31,54-56}$

We use ultrafast transient absorption spectroscopy (TAS) and multi-channel scaling (MCS) PL lifetime analysis for characterizing the photophysics and charge carrier dynamics of pristine and $\mathrm{CdCl}_{2}$ treated PbSe NPLs. TA spectral and time traces of PbSe NPLs with PL centered at $985 \mathrm{~nm}$ (1.3 eV, pristine) and $1050 \mathrm{~nm}\left(1.2 \mathrm{eV}, \mathrm{CdCl}_{2}\right.$ treated) are exemplarily shown in Figure 3. We exclude the formation of an alloy or CdSe shell growth on PbSe NPLs during the metal halide treatment since reaction temperatures of $0{ }^{\circ} \mathrm{C}$ and room temperature are rather low (see Figure 4a for comparison). 56,57

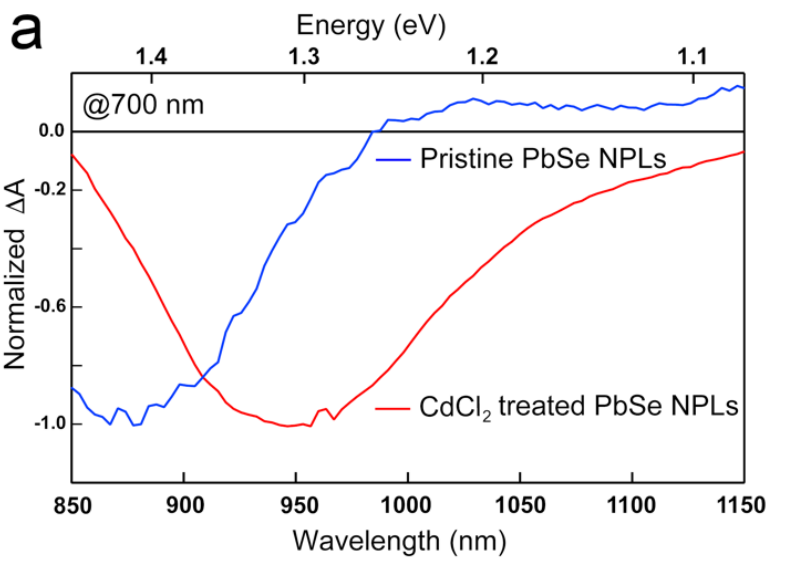

b

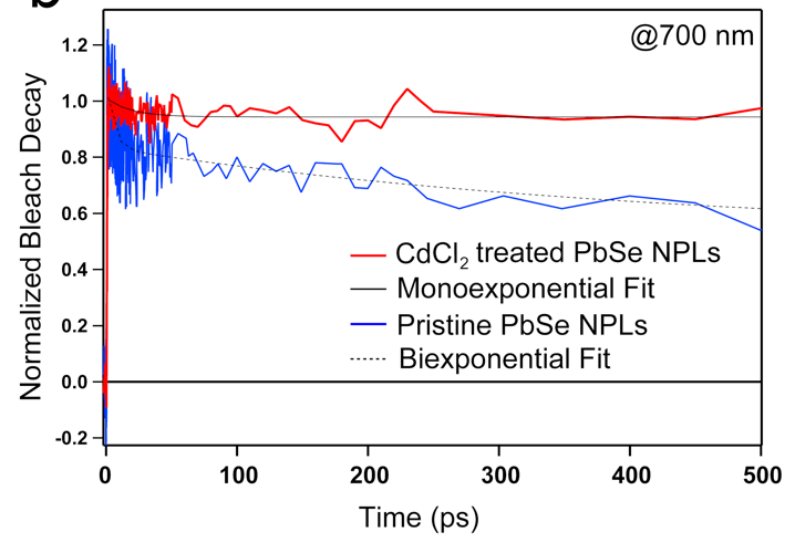

Figure 3: TA spectra of PbSe NPLs emitting at $985 \mathrm{~nm}$ and $1050 \mathrm{~nm}$, resp. and photoexcited at $700 \mathrm{~nm}$. a) Spectral line cuts of pristine (blue) and $\mathrm{CdCl}_{2}$ treated (red) PbSe NPLs at a delay time of $10 \mathrm{ps}$ after photoexcitation with the samples 
exhibiting a prominent bleach feature at $880 \mathrm{~nm}$ (pristine) and $950 \mathrm{~nm}\left(\mathrm{CdCl}_{2}\right.$ treated), resp. b) Exciton dynamics of the pristine (blue) and $\mathrm{CdCl}_{2}$ treated (red) PbSe NPLs show a decay over the course of the measurement with transients of the pristine samples decaying biexponentially, while $\mathrm{CdCl}_{2}$ treated samples exhibit single exponential decay dynamics.

PbSe NPL samples were photoexcited at $700 \mathrm{~nm}$ (with an optical density of sample solutions of $\leq 0.1$ ) and with comparable photoexcitation fluence $\left(30 \mu \mathrm{J} / \mathrm{cm}^{2}\right)$ to ensure a similar distribution of the number of photoexcited excitons in pristine and $\mathrm{CdCl}_{2}$ treated PbSe NPL samples. ${ }^{31}$ TA spectral line cuts at 10 ps after photoexcitation shown in Figure 3a exhibit a prominent ground state bleach (GSB) feature due to state filling and stimulated emission centered at $880 \mathrm{~nm}$ for the pristine, and $950 \mathrm{~nm}$ for the $\mathrm{CdCl}_{2}$ treated NPLs, respectively. 58 Generally, the GSB of PbSe NPLs is slightly blue-shifted with respect to the steady-state absorption by a biexcitonic shift ${ }^{58}$. Due to the initial fast cooling of hot charges (photoexcitation at $700 \mathrm{~nm}$, above the NPL band edge, see Figure S5 for absorption and Figure S6 for color-coded 2D spectra), a short-lived induced absorption is probed, which decays within the first 2 ps of the measurement. After the $\mathrm{CdCl}_{2}$ treatment, the GSB of PbSe NPLs is red-shifted by $70 \mathrm{~nm}(100 \mathrm{meV})$ and hence following the same trend as the steady-state absorption and emission of the NPLs. Spectral line cuts taken at short (1 ps) and longer (3.5 ns) times after photoexcitation (Figure S7) underpin changes in the GSB feature of PbSe NPLs mainly at their emission wavelengths ( $985 \mathrm{~nm}$ and $1050 \mathrm{~nm}$ ) over the course of the measurement. We find the bleach contribution of pristine PbSe NPLs at $985 \mathrm{~nm}$ to have significantly decayed after $3.5 \mathrm{~ns}$, while the $\mathrm{CdCl}_{2}$ treated samples still exhibit a considerably higher contribution at $1050 \mathrm{~nm}$ after $3.5 \mathrm{~ns}$. This is in good agreement with the measured PLQY values of the samples $(2.3 \%$ before and $35.3 \%$ after the $\mathrm{CdCl}_{2}$ treatment). Figure $3 \mathrm{~b}$ depicts the exciton decay dynamics of PbSe NPLs with the GSB features of pristine and $\mathrm{CdCl}_{2}$ treated PbSe NPLs being long-lived generally (exceeding the measurement time window of $6 \mathrm{~ns}$, Figure S6), which is well in accordance with the PLQY found for both samples. The decay dynamics of the pristine PbSe NPLs are fitted biexponentially $\quad\left(\tau_{1 \text { Bleach }}=5.5 \pm 1.5 p s, \quad \tau_{2 \text { Bleach }}=\right.$ $400 \pm 160$ ps), while $\mathrm{CdCl}_{2}$ treated samples can be described with a single exponential decay $\left(\tau_{1 \text { Bleach }}=19 \pm 4 p s\right)$, rendering the treatment efficient for reducing non-radiative recombination in PbS NPLs and leading to their PLQY increase.

\section{PL Contributions}

The absorption and PL spectra of PbSe NPLs and associated TEM images before and after the $\mathrm{CdCl}_{2}$ treatment are shown in Figure 4 (size histograms in Figure S8). We find two components contributing to the PL lifetime, which is apparent from the slightly asymmetric shape of the PbSe NPL PL (Figure 4a) and has been described for PbS and PbSe NCs. ${ }^{59,60}$ We fit the PL spectra with the sum of two Gaussians $31,61,62$ and the Gaussian centered at higher energy (labeled A) is ascribed to the PbSe bandgap associated PL, while the lower energy PL (labeled B) is related to surface traps. ${ }^{6}$

Table 2: PL lifetimes of pristine and $\mathrm{CdCl}_{2}$ treated $\mathrm{PbSe}$ NPLs (emitting at $940 \mathrm{~nm}$ and $980 \mathrm{~nm}$, resp.)

\begin{tabular}{lllll}
\hline $\begin{array}{l}\text { PbSe } \\
\text { NPLs }\end{array}$ & $\begin{array}{l}\tau_{1 P L} \\
(\mathrm{~ns})\end{array}$ & $\mathrm{A}_{1}(\%)$ & $\begin{array}{l}\tau_{2 P L} \\
(\mathrm{~ns})\end{array}$ & $\mathrm{A}_{2}(\%)$ \\
\hline $\begin{array}{l}\text { Pristine } \\
\mathrm{CdCl}_{2}\end{array}$ & 168 & 40 & 1320 & 60 \\
treated & 168 & 15 & 1320 & 85
\end{tabular}

The PL lifetimes of PbSe NPLs (Figure 4b and Table 2) are fitted biexponentially with the same time constants $\tau_{1 P L}=168 \mathrm{~ns}$ and $\tau_{2 P L}=1320 \mathrm{~ns}$. They are longer than lifetimes typically measured for PbSe NCs (100 - $200 \mathrm{~ns})$ and in the same ballpark as lifetimes determined for PbSe NPLs obtained by cation exchange $(0.7-2.7 \mu S) .20,63,64$ For fitting the data, the PL lifetime of the $\mathrm{CdCl}_{2}$ treated PbSe NPLs was run freely, and the pristine sample was fitted using fixed lifetime parameters with both components exhibiting good residua $\left(\mathrm{R}^{2}=0.98\right.$ and $\mathrm{R}^{2}=0.97$, resp.). After the $\mathrm{CdCl}_{2}$ treatment however, we find the contribution of the faster time constant significantly reduced from $40 \%$ to $15 \%$ (Table 2), associated with an increase of the slower time constant to $85 \%$ (Table 2). We assign these contributions to two radiative processes present in the PbSe NPL PL, with the faster time constant related to surface trap state emission and the slower time constant related to exciton recombination. The decrease of the contribution of $\tau_{1 P L}$ leads to an increase in the PL lifetime and PLQY of the PbSe NPLs, rendering the $\mathrm{CdCl}_{2}$ surface treatment successful in fixing surface-related trap states 54,65 To assign the time constants extracted from the PL lifetime measurements to their respective PL contribution, we exemplary determine the PL lifetimes at the maxima of each Gaussian fitted (see Figure S9 and Table S1) and find a biexponential decay, with different contributions of the two constants to the respective PL decay. The shorter PL lifetime constant $\left(\tau_{1 P L}=168 \mathrm{~ns}\right)$ shows higher contribution at the maximum of peak $B$ and is thus assigned to the trap state related emission, while the longer PL lifetime constant $\left(\tau_{2 P L}=1320 \mathrm{~ns}\right)$ is dominant at peak $\mathrm{A}$ and is assigned to the excitonic emission. Even for pristine PbSe NPLs, the trap state emission shows a small contribution, while the PL is dominated by the bandgap transition (ratio A:B 6.5:1). After the $\mathrm{CdCl}_{2}$ treatment, this effect is further enhanced as is shown by the peak area ratio of feature A (ratio A:B increasing to 22:1, see Table 3).

The FWHM of the excitonic PL peak A decreases slightly from 221 to $214 \mathrm{meV}$ after the $\mathrm{CdCl}_{2}$ treatment, while the FWHM of the PL peak B is significantly reduced from $217 \mathrm{meV}$ to $149 \mathrm{meV}$ (Figure 4a) further underpinning the positive effect of the $\mathrm{CdCl}_{2}$ treatment. All PbSe NPL samples synthesized in this work follow this trend, and a FWHM of $180-220 \mathrm{meV}$ is representative for the NPL PL, independent of the respective PL maximum (Table S2). The FWHM of PbSe NPLs synthesized here are slightly broader than FWHM typically obtained for PbSe NCs $(50-150 \mathrm{meV})$ that exhibit a bandgap below $1.5 \mathrm{eV}$ as well as PbSe NPLs synthesized by cation exchange (PL at $0.93 \mathrm{eV}$, FWHM of $116 \mathrm{meV}$ for contribution A). ${ }^{20,62,66}$ We attribute the PL FWHM values of the PbSe NPLs shown here mainly to their lateral size distribution. However, $\mathrm{PbS}$ and $\mathrm{PbSe} \mathrm{NCs}$ generally exhibit tunable emission with decreasing PLQY values for increasing NC sizes. ${ }^{67}$ For example, while the PLQY for small PbSe NCs with a bandgap $>1.3 \mathrm{eV}$ reaches $60 \%$, NCs with a bandgap $<1.2 \mathrm{eV}$ have a PLQY of less than $40 \% .66,67$ This renders the PbSe NPLs shown here superior at shorter wavelengths (higher energy) where their PLQY values match or even exceed the PLQY of small PbSe NCs. 

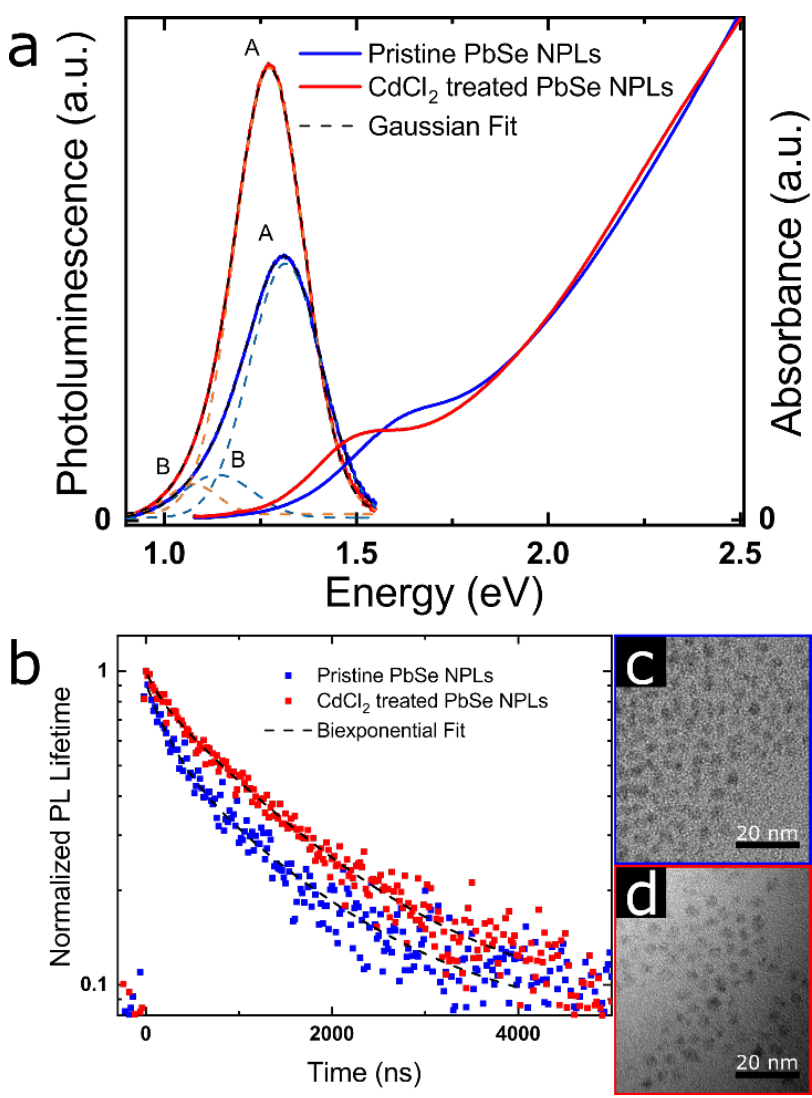

Figure 4: PbSe NPL PL properties (emitting at $940 \mathrm{~nm}$ [pristine] and $980 \mathrm{~nm}\left[\mathrm{CdCl}_{2}\right.$ treated]). a) PL and absorbance. The PL data was corrected for the transformation from the wavelength to the energy scale by a Jacobian transformation. ${ }^{68}$ The dashed lines represent a fit of the PL with the sum of two Gaussians. b) MCS PL lifetime measurements, taken at each PL maximum and exhibiting a single exponential decay with increased lifetimes after the $\mathrm{CdCl}_{2}$ treatment. c) and d) TEM images of the pristine and $\mathrm{CdCl}_{2}$ treated NPLs showing a slight NPL size increase after the treatment.

Table 3: Values for the two Gaussians of pristine and $\mathrm{CdCl}_{2}$ treated PbSe NPLs (emitting at $940 \mathrm{~nm}$ and $980 \mathrm{~nm}$, resp.)

\begin{tabular}{lccccccc}
\multirow{2}{*}{$\begin{array}{c}\text { PbSe } \\
\text { NPLs }\end{array}$} & $\begin{array}{c}\text { peak center } \\
(\mathrm{eV})\end{array}$ & \multicolumn{2}{c}{$\begin{array}{c}\text { FWHM } \\
(\mathrm{meV})\end{array}$} & peak area & $\begin{array}{c}\text { peak } \\
\text { ratio }\end{array}$ \\
\cline { 2 - 8 } & $\mathrm{A}$ & $\mathrm{B}$ & $\mathrm{A}$ & $\mathrm{B}$ & $\mathrm{A}$ & $\mathrm{B}$ & $\mathrm{A}: \mathrm{B}$ \\
\hline pristine & 1.32 & 1.14 & 221 & 217 & 0.13 & 0.02 & 6.5 \\
$\mathrm{CdCl}_{2}$ & 1.27 & 1.07 & 214 & 149 & 0.22 & 0.01 & 22.0 \\
treated & & & & & & &
\end{tabular}

TEM images shown in Figure $4 \mathrm{c}$ and $4 \mathrm{~d}$ (as well as Figure 1) underpin the lack of a NPL shape transformation upon $\mathrm{CdCl}_{2}$ treatment. A small lateral size increase (from $3.6 \times 3.1 \mathrm{~nm}^{2}$ to $4.2 \times 3.5 \mathrm{~nm}^{2}$ ) gets apparent, which is also shown in the redshift of the NPL absorption after the treatment. The lateral size most likely originates from the additional reaction time after the $\mathrm{CdCl}_{2}$ treatment of the pristine NPLs. However, a change in the thickness of the NPLs under $\mathrm{CdCl}_{2}$ treatment cannot be ruled out completely and has been e.g. observed for CdSe NPLs.69-71 The $\mathrm{CdCl}_{2}$ treatment of PbSe NPLs is accompanied by a significant increase of the PLQY with the highest value determined for
NPLs with an emission maximum of $1.27 \mathrm{eV}(980 \mathrm{~nm})$ from $14.7 \%$ before to $37.4 \%$. Excitation spectra shown in Figure S10 additionally support the absence of a contribution of spherical PbSe NCs as side products in the synthesis to the NPL PL.

The chemical composition of the PbSe NPLs was determined by EDXS (see Table S3). We find that the NPLs exhibit a slight Pb excess before and after the $\mathrm{CdCl}_{2}$ treatment, which is typical for $\mathrm{PbSe}$ NCs (Pb:Se ratio 1.2:1).72 The optical properties of PbSe NPLs shown in Figure 4c and 4d only indicate small changes in the NPL lateral size, which is supported by the TEM images. Furthermore, after the $\mathrm{CdCl}_{2}$ treatment EDXS results show a $\mathrm{Cd}: \mathrm{Cl}$ ratio of 1.8:1, closely resembling the molecular ratio of $\mathrm{CdCl}_{2}$. This indicates $\mathrm{CdCl}_{2}$ acting as Z-type ligand for stabilizing PbSe NPLs. ${ }^{43}$ However, chloride anions could also act as X-type ligands, providing charge neutrality to the NPLs. 54,55

By assuming an inversely linear relationship between the PLQY and the number of trap states in the PbSe NPLs, i.e. halving the number of trap states results in a doubling of the PLQY, we estimate the fixing of surface related traps by treating the $\mathrm{PbSe}$ NPLs with $\mathrm{CdCl}_{2}$ and conclude that nearly half of the initial surface traps in pristine NPLs are saturated by the $\mathrm{CdCl}_{2}$ treatment. ${ }^{4}$ With larger NPLs, we consider if a single surface related trap is sufficient to quench the NPL PL or if there is a difference between single and multiple trap states per NPL ${ }^{73}$ and incorporate the hyperbolic proportionality between the bandgap and the NPL edge length shown in Figure 2a.

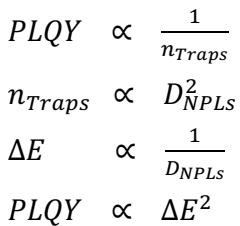

We find a parabolic proportionality between the PLQY and the PL position (see Figure S11) of PbSe NPLs underpinning that either a single trap per NPL occurs or that these single traps lack to quench the bandgap emission completely. For further increasing the PLQY of PbSe NPLs, elaborate surface treatments are currently investigated.

\section{Conclusion}

In conclusion, we have shown the first low temperature direct colloidal synthesis of PbSe NPLs with distinct excitonic absorption features in the range of $800-1000 \mathrm{~nm}(1.6-1.3 \mathrm{eV})$. The optical properties of the NPLs are tuned by controlling their lateral dimension with the addition of octylamine and the reaction temperature, with increasing octylamine amount and higher reaction temperatures yielding larger NPLs. The efficient infrared PL of PbSe NPLs with QY values of up to $37.4 \%$ between $900-1450 \mathrm{~nm}(1.4-0.9 \mathrm{eV})$ is obtained by their postsynthetic treatment with $\mathrm{CdCl}_{2}$. Transient absorption and time-resolved PL spectroscopy reveal a considerable decrease of surface related trap states and non-radiative recombination in the $\mathrm{PbSe}$ NPLs by the treatment, rendering them efficient colloidal quantum emitters at telecommunication wavelengths.

\section{ASSOCIATED CONTENT}

SI: This material is available free of charge via the Internet at http://pubs.acs.org.

\section{AUTHOR INFORMATION}

\section{Corresponding Author}

* jannika.lauth@uni-hannover.de 


\section{Author Contributions}

The manuscript was written through contributions of all authors. All authors have given approval to the final version of the manuscript.

\section{Funding Sources}

The project leading to these results was funded by the Deutsche Forschungsgemeinschaft (DFG, German Research Foundation) under Germany's Excellence Strategy within the Cluster of Excellence PhoenixD (EXC 2122, Project ID 390833453). J.L. is thankful for additional funding by the Caroline Herschel program of the Leibniz Universität Hannover.

\section{ACKNOWLEDGMENT}

The authors thank the Laboratory for Nano and Quantum Engineering (LNQE) in Hannover for access to the TEM. We thank Prof. Nadja-C. Bigall for access to the photoluminescence spectrometer and Prof. Dirk Dorfs for access to the UV-Vis-NIR absorption spectrometer. We are grateful to Prof. Armin Feldhoff for providing the XRD facilities.

\section{ABBREVIATIONS}

EDXS, energy dispersive X-ray spectroscopy; FWHM, full width at half maximum; GSB, ground state bleach; Hex, hexane; HOA, oleic acid; ML, mono layer; NCs, nanocrystals; NIR, near infrared; NPLs, nanoplatelets; NSs, nanosheets; OcAm, Octylamine; PL, photoluminescence; PLQY, photoluminescence quantum yield; SWIR, short-wave-infrared; TAS, transient absorption spectroscopy; TEM, transmission electron microscopy; TCE, tetrachloroethylene; XRD, X-ray diffraction

\section{REFERENCES}

(1) Schliehe, C.; Juarez, B. H.; Pelletier, M.; Jander, S.; Greshnykh, D.; Nagel, M.; Meyer, A.; Foerster, S.; Kornowski, A.; Klinke, C.; Weller, H. Ultrathin PbS Sheets by Two-Dimensional Oriented Attachment. Science 2010, 329, 550-553.

(2) Akkerman, Q. A.; Martín-García, B.; Buha, J.; Almeida, G.; Toso, S.; Marras, S.; Bonaccorso, F.; Petralanda, U.; Infante, I.; Manna, L. Ultrathin Orthorhombic PbS Nanosheets. Chem. Mater. 2019, 31, 8145-8153.

(3) Koh, W.-K.; Dandu, N. K.; Fidler, A. F.; Klimov, V. I.; Pietryga, J. M.; Kilina, S. V. Thickness-Controlled Quasi-Two-Dimensional Colloidal PbSe Nanoplatelets. J. Am. Chem. Soc. 2017, 139, 2152-2155.

(4) Jiang, Z.; Bhandari, G. B.; Premathilaka, S. M.; Khan, S.; Dimick, D. M.; Stombaugh, C.; Mandell, A.; He, Y.; Peter Lu, H.; Sun, L. Growth of Colloidal PbS Nanosheets and the Enhancement of their Photoluminescence. Phys. Chem. Chem. Phys. 2015, 17, 23303-23307.

(5) Desmet, K. D.; Paz, D. A.; Corry, J. J.; Eells, J. T.; Wong-Riley, M. T. T.; Henry, M. M.; Buchmann, E. V.; Connelly, M. P.; Dovi, J. V.; Liang, H. L.; Henshel, D. S.; Yeager, R. L.; Millsap, D. S.; Lim, J.; Gould, L. J.; Das, R.; Jett, M.; Hodgson, B. D.; Margolis, D.; Whelan, H. T. Clinical and Experimental Applications of NIR-LED Photobiomodulation. Photomed. Laser Surg. 2006, 24, 121-128.

(6) Koch, F.; Chernikov, S. V.; Taylor, J. R. Dispersion Measurement in Optical Fibres Over the Entire Spectral Range from 1.1 $\mu \mathrm{m}$ to $1.7 \mu \mathrm{m}$. Opt. Commun. 2000, 175, 209-213.

(7) Moayed, M. M. R.; Bielewicz, T.; Zoellner, M. S.; Herrmann, C.; Klinke, C. Towards Colloidal Spintronics through Rashba Spin-Orbit Interaction in Lead Sulphide Nanosheets. Nat. Commun. 2017, 8, 15721.
(8) Aerts, M.; Bielewicz, T.; Klinke, C.; Grozema, F. C.; Houtepen, A. J.; Schins, J. M.; Siebbeles, L. D. A. Highly Efficient Carrier Multiplication in PbS Nanosheets. Nat. Commun. 2014, 5, 3789.

(9) Dogan, S.; Bielewicz, T.; Cai, Y.; Klinke, C. Field-Effect Transistors Made of Individual Colloidal PbS Nanosheets. Appl. Phys. Lett. 2012, 101, 73102.

(10) Lauth, J.; Failla, M.; Klein, E.; Klinke, C.; Kinge, S.; Siebbeles, L. D. A. Photoexcitation of PbS Nanosheets Leads to Highly Mobile Charge Carriers and Stable Excitons. Nanoscale 2019, 11, 21569-21576.

(11) Bielewicz, T.; Dogan, S.; Klinke, C. Tailoring the Height of Ultrathin PbS Nanosheets and Their Application as Field-Effect Transistors. Small 2015, 11, 826-833.

(12) Chen, Z.; Nadal, B.; Mahler, B.; Aubin, H.; Dubertret, B. Quasi-2D Colloidal Semiconductor Nanoplatelets for Narrow Electroluminescence. Adv. Funct. Mater. 2014, 24, 295-302.

(13) Bernardi, M.; Palummo, M.; Grossman, J. C. Extraordinary Sunlight Absorption and One Nanometer Thick Photovoltaics Using Two-Dimensional Monolayer Materials. Nano Lett 2013, 13, 3664-3670.

(14) Dogan, S.; Bielewicz, T.; Lebedeva, V.; Klinke, C. Photovoltaic Effect in Individual Asymmetrically Contacted Lead Sulfide Nanosheets. Nanoscale 2015, 7, 4875-4883.

(15) Cao, X.; Zopf, M.; Ding, F. Telecom Wavelength Single Photon Sources. J. Semicond. 2019, 40, 71901.

(16) Krishnamurthy, S.; Singh, A.; Hu, Z.; Blake, A. V.; Kim, Y.; Singh, A.; Dolgopolova, E. A.; Williams, D. J.; Piryatinski, A.; Malko, A. V.; Htoon, H.; Sykora, M.; Hollingsworth, J. A. PbS/CdS Quantum Dot Room-Temperature Single-Emitter Spectroscopy Reaches the Telecom 0 and S Bands via an Engineered Stability. ACS Nano 2021, 15, 575-587.

(17) Hanson, C. J.; Hartmann, N. F.; Singh, A.; Ma, X.; DeBenedetti, W. J. I.; Casson, J. L.; Grey, J. K.; Chabal, Y. J.; Malko, A. V.; Sykora, M.; Piryatinski, A.; Htoon, H.; Hollingsworth, J. A. Giant $\mathrm{PbSe} / \mathrm{CdSe} / \mathrm{CdSe}$ Quantum Dots: Crystal-Structure-Defined Ultrastable Near-Infrared Photoluminescence from Single Nanocrystals. J. Am. Chem. Soc. 2017, 139, 11081-11088.

(18) Xu, F.; Ma, X.; Zhang, Q.; Lo, H.-K.; Pan, J.-W. Secure Quantum Key Distribution with Realistic Devices. Rev. Mod. Phys. 2020, 92, 025002.

(19) Tenney, S. M.; Vilchez, V.; Sonnleitner, M. L.; Huang, C.; Friedman, H. C.; Shin, A. J.; Atallah, T. L.; Deshmukh, A. P.; Ithurria, S.; Caram, J. R. Mercury Chalcogenide Nanoplatelet-Quantum Dot Heterostructures as a New Class of Continuously Tunable Bright Shortwave Infrared Emitters. J. Phys. Chem. Lett. 2020, 11, 3473-3480.

(20) Galle, T.; Samadi Khoshkhoo, M.; Martin-Garcia, B.; Meerbach, C.; Sayevich, V.; Koitzsch, A.; Lesnyak, V.; Eychmüller, A. Colloidal PbSe Nanoplatelets of Varied Thickness with Tunable Optical Properties. Chem. Mater. 2019, 31, 3803-3811.

(21) Galle, T.; Spittel, D.; Weiß, N.; Shamraienko, V.; Decker, H.; Georgi, M.; Hübner, R.; Metzkow, N.; Steinbach, C.; Schwarz, D.; Lesnyak, V.; Eychmüller, A. Simultaneous Ligand and Cation Exchange of Colloidal CdSe Nanoplatelets toward PbSe Nanoplatelets for Application in Photodetectors. J. Phys. Chem. Lett. 2021, 12, 5214-5220.

(22) Ithurria, S.; Tessier, M. D.; Mahler, B.; Lobo, R. P. S. M.; Dubertret, B.; Efros, A. L. Colloidal Nanoplatelets with Two-Dimensional Electronic Structure. Nat. Mater. 2011, 10, 936-941.

(23) Khan, A. H.; Brescia, R.; Polovitsyn, A.; Angeloni, I.; MartínGarcía, B.; Moreels, I. Near-Infrared Emitting Colloidal PbS Nanoplatelets: Lateral Size Control and Optical Spectroscopy. Chem. Mater. 2017, 29, 2883--2889.

(24) Khan, A. H.; Pal, S.; Dalui, A.; Pradhan, J.; Sarma, D. D.; Acharya, S. Solution-Processed Free-Standing Ultrathin Two- 
Dimensional PbS Nanocrystals with Efficient and Highly Stable Dielectric Properties. Chem. Mater. 2017, 29, 1175-1182.

(25) Antu, A. D.; Jiang, Z.; Premathilka, S. M.; Tang, Y.; Hu, J.; Roy, A.; Sun, L. Bright Colloidal PbS Nanoribbons. Chem. Mater. 2018, 30, 3697-3703.

(26) Zhang, H.; Savitzky, B. H.; Yang, J.; Newman, J. T.; Perez, K. A.; Hyun, B.-R.; Kourkoutis, L. F.; Hanrath, T.; Wise, F. W. Colloidal Synthesis of $\mathrm{PbS}$ and $\mathrm{PbS} / \mathrm{CdS}$ Nanosheets Using AcetateFree Precursors. Chem. Mater. 2016, 28, 127-134.

(27) Vaughn, D. D.; Patel, R. J.; Hickner, M. A.; Schaak, R. E. Single-Crystal Colloidal Nanosheets of GeS and GeSe. J. Am. Chem. Soc. 2010, 132, 15170-15172.

(28) Morrison, P. J.; Loomis, R. A.; Buhro, W. E. Synthesis and Growth Mechanism of Lead Sulfide Quantum Platelets in Lamellar Mesophase Templates. Chem. Mater. 2014, 26, 50125019.

(29) Campos, M. P.; Hendricks, M. P.; Beecher, A. N.; Walravens, W.; Swain, R. A.; Cleveland, G. T.; Hens, Z.; Sfeir, M. Y.; Owen, J. S. A Library of Selenourea Precursors to PbSe Nanocrystals with Size Distributions near the Homogeneous Limit. J. Am. Chem. Soc. 2017, 139, 2296-2305.

(30) Owen, J. S.; Chan, E. M.; Liu, H.; Alivisatos, A. P. Precursor Conversion Kinetics and the Nucleation of Cadmium Selenide Nanocrystals. J. Am. Chem. Soc. 2010, 132, 18206-18213.

(31) Manteiga Vázquez, F.; Yu, Q.; Klepzig, L. F.; Siebbeles, L. D. A.; Crisp, R. W.; Lauth, J. Probing Excitons in Ultrathin PbS Nanoplatelets with Enhanced Near-Infrared Emission. J. Phys. Chem. Lett. 2021, 12, 680-685.

(32) Chen, Y.; Chen, D.; Li, Z.; Peng, X. Symmetry-Breaking for Formation of Rectangular CdSe Two-Dimensional Nanocrystals in Zinc-Blende Structure. J. Am. Chem. Soc. 2017, 139, 1000910019.

(33) Zhou, Y.; Celikin, M.; Camellini, A.; Sirigu, G.; Tong, X.; Jin, L.; Basu, K.; Barba, D.; Ma, D.; Sun, S.; Vidal, F.; Zavelani-Rossi, M.; Wang, Z. M.; Zhao, H.; Vomiero, A.; Rosei, F. Ultrasmall Nanoplatelets: The Ultimate Tuning of Optoelectronic Properties. Adv. Energy Mater. 2017, 7, 1602728.

(34) Liu, Y.-H.; Wang, F.; Wang, Y.; Gibbons, P. C.; Buhro, W. E. Lamellar assembly of cadmium selenide nanoclusters into quantum belts. J. Am. Chem. Soc. 2011, 133, 17005-17013.

(35) Son, J. S.; Wen, X.-D.; Joo, J.; Chae, J.; Baek, S.-I.; Park, K.; Kim, J. H.; An, K.; Yu, J. H.; Kwon, S. G.; Choi, S.-H.; Wang, Z.; Kim, Y.-W.; Kuk, Y.; Hoffmann, R.; Hyeon, T. Large-Scale Soft Colloidal Template Synthesis of $1.4 \mathrm{~nm}$ Thick CdSe Nanosheets. Angew. Chem. Int. Ed. 2009, 48, 6861-6864.

(36) Riedinger, A.; Ott, F. D.; Mule, A.; Mazzotti, S.; Knüsel, P. N.; Kress, S. J.; Prins, F.; Erwin, S. C.; Norris, D. J. An intrinsic Growth Instability in Isotropic Materials Leads to Quasi-Two-Dimensional Nanoplatelets. Nat. Mater. 2017, 16, 743-748.

(37) Almeida, G.; Goldoni, L.; Akkerman, Q.; Dang, Z.; Khan, A. H.; Marras, S.; Moreels, I.; Manna, L. Role of Acid-Base Equilibria in the Size, Shape, and Phase Control of Cesium Lead Bromide Nanocrystals. ACS Nano 2018, 12, 1704-1711.

(38) Hendricks, M. P.; Campos, M. P.; Cleveland, G. T.; Jen-La Plante, I.; Owen, J. S. A Tunable Library of Substituted Thiourea Precursors to Metal Sulfide Nanocrystals. Science 2015, 348, 1226-1230.

(39) Lauth, J.; Grimaldi, G.; Kinge, S.; Houtepen, A. J.; Siebbeles, L. D. A.; Scheele, M. Ultrafast Charge Transfer and Upconversion in Zinc $\beta$-Tetraaminophthalocyanine-Functionalized $\mathrm{PbS}$ Nanostructures Probed by Transient Absorption Spectroscopy. Angew. Chem. Int. Ed. 2017, 56, 14061-14065.

(40) Spoor, F. C. M.; Kunneman, L. T.; Evers, W. H.; Renaud, N.; Grozema, F. C.; Houtepen, A. J.; Siebbeles, L. D. A. Hole Cooling Is
Much Faster than Electron Cooling in PbSe Quantum Dots. ACS Nano 2016, 10, 695-703.

(41) McGuire, J. A.; Joo, J.; Pietryga, J. M.; Schaller, R. D.; Klimov, V. I. New Aspects of Carrier Multiplication in Semiconductor Nanocrystals. Acc. Chem. Res. 2008, 41, 1810-1819.

(42) Wang, Y.; Zhou, Y.; Zhang, Y.; Buhro, W. E. Magic-Size II-VI Nanoclusters as Synthons for Flat Colloidal Nanocrystals. Inorg. Chem. 2015, 54, 1165-1177.

(43) Ekimov, A. I.; Onushchenko, A. A. Quantum Size Effect in Three-Dimensional Microscopic Semiconductor Crystals. J. Exp. Theor. Phys. Lett. 1981, 34, 345-348.

(44) Dai, Q.; Wang, Y.; Li, X.; Zhang, Y.; Pellegrino, D. J.; Zhao, M.; Zou, B.; Seo, J.; Wang, Y.; Yu, W. W. Size-Dependent Composition and Molar Extinction Coefficient of PbSe Semiconductor Nanocrystals. ACS Nano 2009, 3, 1518-1524.

(45) Kang, I.; Wise, F. W. Electronic Structure and Optical Properties of PbS and PbSe Quantum Dots. J. Opt. Soc. Am. B 1997, 14,1632 .

(46) Allan, G.; Delerue, C. Unusual Quantum Confinement Effects in IV-VI Materials. Mater. Sci. Eng. C. 2005, 25, 687-690.

(47) Spoor, F. C. M.; Grimaldi, G.; Delerue, C.; Evers, W. H.; Crisp, R. W.; Geiregat, P.; Hens, Z.; Houtepen, A. J.; Siebbeles, L. D. A. Asymmetric Optical Transitions Determine the Onset of Carrier Multiplication in Lead Chalcogenide Quantum Confined and Bulk Crystals. ACS Nano 2018, 12, 4796-4802.

(48) Wang, Y.; Suna, A.; Mahler, W.; Kasowski, R. PbS in polymers. From Molecules to Bulk Solids. J. Chem. Phys. 1987, 87, 7315-7322.

(49) Lead selenide (PbSe) Energy Gap and Band Structure. In Non-Tetrahedrally Bonded Elements and Binary Compounds I; Madelung, O., Rössler, U., Schulz, M., Eds.; Landolt-Börnstein Group III Condensed Matter; Springer-Verlag, 1998; pp 1-8. DOI: $10.1007 / 10681727 \_890$.

(50) Ithurria, S.; Bousquet, G.; Dubertret, B. Continuous Transition from 3D to 1D Confinement Observed During the Formation of CdSe Nanoplatelets. J. Am. Chem. Soc. 2011, 133, 3070-3077.

(51) Bertrand, G. H. V.; Polovitsyn, A.; Christodoulou, S.; Khan, A. H.; Moreels, I. Shape Control of Zincblende CdSe Nanoplatelets. Chem Commun. 2016, 52, 11975-11978.

(52) Brus, L. Electronic Wave Functions in Semiconductor Clusters: Experiment and Theory. J. Phys. Chem. 1986, 90, 2555-2560.

(53) Kunneman, L. T.; Schins, J. M.; Pedetti, S.; Heuclin, H.; Grozema, F. C.; Houtepen, A. J.; Dubertret, B.; Siebbeles, L. D. A. Nature and Decay Pathways of Photoexcited States in CdSe and CdSe/CdS Nanoplatelets. Nano Lett. 2014, 14, 7039-7045.

(54) Kirkwood, N.; Monchen, J. O. V.; Crisp, R. W.; Grimaldi, G.; Bergstein, H. A. C.; Du Fossé, I.; van der Stam, W.; Infante, I.; Houtepen, A. J. Finding and Fixing Traps in II-VI and III-V Colloidal Quantum Dots: The Importance of Z-Type Ligand Passivation. J. Am. Chem. Soc. 2018, 140, 15712-15723.

(55) Dufour, M.; Qu, J.; Greboval, C.; Méthivier, C.; Lhuillier, E.; Ithurria, S. Halide Ligands To Release Strain in Cadmium Chalcogenide Nanoplatelets and Achieve High Brightness. ACS Nano 2019, 13, 5326-5334.

(56) Bae, W. K.; Joo, J.; Padilha, L. A.; Won, J.; Lee, D. C.; Lin, Q.; Koh, W.-K.; Luo, H.; Klimov, V. I.; Pietryga, J. M. Highly Effective Surface Passivation of PbSe Quantum Dots through Reaction with Molecular Chlorine. J. Am. Chem. Soc. 2012, 134, 20160 20168.

(57) Green, P. B.; Li, Z.; Wilson, M. W. PbS Nanocrystals Made with Excess $\mathrm{PbCl}_{2}$ Have an Intrinsic Shell that Reduces Their Stokes Shift. J. Phys. Chem. Lett. 2019, 10, 5897-5901. 
(58) Schins, J. M.; Trinh, M. T.; Houtepen, A. J.; Siebbeles, L. D. A. Probing Formally Forbidden Optical Transitions in PbSe Nanocrystals by Time- and Energy-Resolved Transient Absorption Spectroscopy. Phys. Rev. B 2009, 80 .

(59) Harbold, J. M.; Wise, F. W. Photoluminescence Spectroscopy of PbSe Nanocrystals. Phys. Rev. B 2007, 76.

(60) Caram, J. R.; Bertram, S. N.; Utzat, H.; Hess, W. R.; Carr, J. A.; Bischof, T. S.; Beyler, A. P.; Wilson, M. W. B.; Bawendi, M. G. PbS Nanocrystal Emission Is Governed by Multiple Emissive States. Nano Lett 2016, 16, 6070-6077.

(61) Erdem, O.; Olutas, M.; Guzelturk, B.; Kelestemur, Y.; Demir, H. V. Temperature-Dependent Emission Kinetics of Colloidal Semiconductor Nanoplatelets Strongly Modified by Stacking. J. Phys. Chem. Lett. 2016, 7, 548-554.

(62) Skurlov, I.; Sokolova, A.; Galle, T.; Cherevkov, S.; Ushakova, E.; Baranov, A.; Lesnyak, V.; Fedorov, A.; Litvin, A. TemperatureDependent Photoluminescent Properties of PbSe Nanoplatelets. Nanomaterials. 2020, 10, 2570.

(63) An, J. M.; Franceschetti, A.; Zunger, A. The Excitonic Exchange Splitting and Radiative Lifetime in PbSe Quantum Dots. Nano Lett. 2007, 7, 2129-2135.

(64) Sykora, M.; Koposov, A. Y.; McGuire, J. A.; Schulze, R. K.; Tretiak, O.; Pietryga, J. M.; Klimov, V. I. Effect of Air Exposure on Surface Properties, Electronic Structure, and Carrier Relaxation in PbSe Nanocrystals. ACS Nano 2010, 4, 2021-2034.

(65) Fisher, B. R.; Eisler, H.-J.; Stott, N. E.; Bawendi, M. G. Emission Intensity Dependence and Single-Exponential Behavior In Single Colloidal Quantum Dot Fluorescence Lifetimes. J. Phys. Chem. B 2004, 108, 143-148.

(66) Lu, H.; Carroll, G. M.; Neale, N. R.; Beard, M. C. Infrared Quantum Dots: Progress, Challenges, and Opportunities. ACS Nano 2019, 13, 939-953.
(67) Semonin, O. E.; Johnson, J. C.; Luther, J. M.; Midgett, A. G.; Nozik, A. J.; Beard, M. C. Absolute Photoluminescence Quantum Yields of IR-26 Dye, PbS, and PbSe Quantum Dots. J. Phys. Chem. Lett. 2010, 1, 2445-2450.

(68) Mooney, J.; Kambhampati, P. Get the Basics Right: Jacobian Conversion of Wavelength and Energy Scales for Quantitative Analysis of Emission Spectra. J. Phys. Chem. Lett. 2013, 4, 33163318.

(69) Moghaddam, N.; Dabard, C.; Dufour, M.; Po, H.; Xu, X.; Pons, T.; Lhuillier, E.; Ithurria, S. Surface Modification of CdE (E: S, Se, and Te) Nanoplatelets to Reach Thicker Nanoplatelets and Homostructures with Confinement-Induced Intraparticle Type I Energy Level Alignment. J. Am. Chem. Soc. 2021, 143, 18631872.

(70) Christodoulou, S.; Climente, J. I.; Planelles, J.; Brescia, R.; Prato, M.; Martín-García, B.; Khan, A. H.; Moreels, I. Chloride-Induced Thickness Control in CdSe Nanoplatelets. Nano Lett. 2018, 18, 6248-6254.

(71) Meerbach, C.; Wu, C.; Erwin, S. C.; Dang, Z.; Prudnikau, A.; Lesnyak, V. Halide-Assisted Synthesis of Cadmium Chalcogenide Nanoplatelets. Chem. Mater. 2020, 32, 566-574.

(72) Weidman, M. C.; Beck, M. E.; Hoffman, R. S.; Prins, F.; Tisdale, W. A. Monodisperse, Air-Stable PbS Nanocrystals via Precursor Stoichiometry Control. ACS Nano 2014, 8, 6363-6371.

(73) Munro, A. M.; Jen-La Plante, I.; Ng, M. S.; Ginger, D. S. Quantitative Study of the Effects of Surface Ligand Concentration on CdSe Nanocrystal Photoluminescence. J. Phys. Chem. C 2007, $111,6220-6227$. 